\title{
Review Article \\ Treatments for Neurological Gait and Balance Disturbance: The Use of Noninvasive Electrical Brain Stimulation
}

\author{
Diego Kaski and Adolfo M. Bronstein \\ Division of Brain Sciences, Imperial College London, Charing Cross Hospital, London W6 8RF, UK \\ Correspondence should be addressed to Diego Kaski; d.kaski@imperial.ac.uk
}

Received 11 June 2014; Revised 13 October 2014; Accepted 23 October 2014; Published 3 December 2014

Academic Editor: Paul Sauseng

Copyright (C) 2014 D. Kaski and A. M. Bronstein. This is an open access article distributed under the Creative Commons Attribution License, which permits unrestricted use, distribution, and reproduction in any medium, provided the original work is properly cited.

\begin{abstract}
Neurological gait disorders are a common cause of falls, morbidity, and mortality, particularly amongst the elderly. Neurological gait and balance impairment has, however, proved notoriously difficult to treat. The following review discusses some of the first experiments to modulate gait and balance in healthy adults using anodal transcranial direct current stimulation (tDCS) by stimulating both cerebral hemispheres simultaneously. We review and discuss published data using this novel tDCS approach, in combination with physical therapy, to treat locomotor and balance disorders in patients with small vessel disease (leukoaraiosis) and Parkinson's disease. Finally, we review the use of bihemispheric anodal tDCS to treat gait impairment in patients with stroke in the subacute phase. The findings of these studies suggest that noninvasive electrical stimulation techniques may be a useful adjunct to physical therapy in patients with neurological gait disorders, but further mutlicentre randomized sham-controlled studies are needed to evaluate whether experimental tDCS use can translate into mainstream clinical practice for the treatment of neurological gait disorders.
\end{abstract}

\section{Introduction}

Disorders of gait are a common presentation in neurological practice and general medical settings, particularly amongst the elderly population. In addition, impairment of postural and righting reactions commonly causes falls when turning or bending over [1] leading to insecure walking and the development of a fear of falling [2]. The following review discusses some of the first ever experiments to modulate gait and balance in healthy adults using anodal transcranial direct current stimulation (tDCS) by stimulating both hemispheres simultaneously (Figure 1) and to treat locomotor and balance disorders in patients with neurological gait disturbance.

We show in a series of previously published work that bihemispheric electrical stimulation with a novel montage can increase cortical excitability of lower limb muscles and increases locomotor learning in healthy subjects. The same electrode montage increases gait speed and improves balance in patients with Parkinson's disease and gait disturbance associated with small vessel disease, when combined with physical therapy (Figure 3). Lastly, by increasing excitability in the affected hemisphere and inhibiting the unaffected one, we were able to improve walking time in patients with stroke in the subacute phase, where the potential to modulate cortical excitability is maximal.

\section{Gait Modulation for Neurological Disease}

Gait and balance disturbance is common, difficult to treat despite rehabilitation, and a cause of significant morbidity and mortality. Parkinson's disease is a common cause of gait and postural instability and although it is defined pathologically by the loss of dopaminergic neurons in the substantia nigra, much of the long-term disability is related to symptoms that do not respond to levodopa [3]. We therefore sought to apply noninvasive brain stimulation techniques using a novel electrode montage in patients with Parkinson's disease to improve gait and balance.

In Parkinson's disease, an overactivity of inhibitory efferents from basal ganglia to the thalamus results in suppression on thalamocortical projections, with reduced activity in premotor and primary motor cortical regions $[4,5]$. The motor cortex may thus be an important therapeutic neurostimulatory target in these patients. 


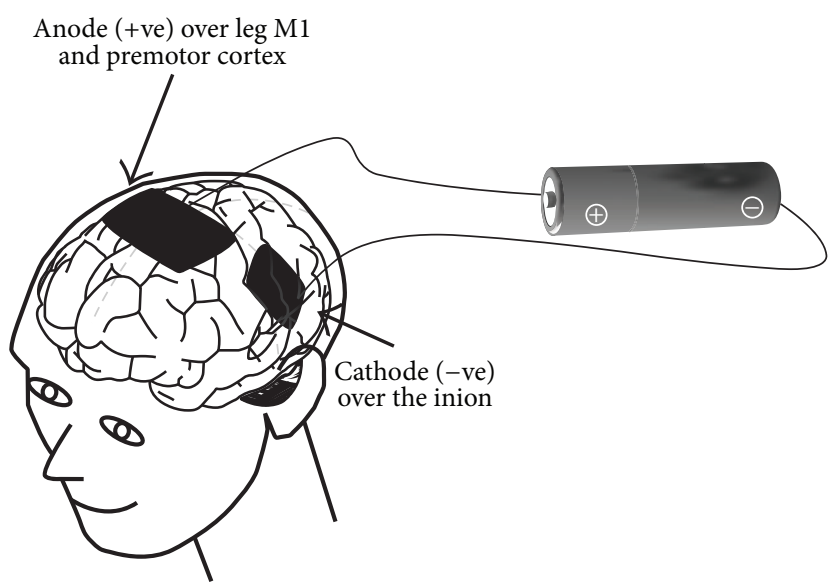

(a)

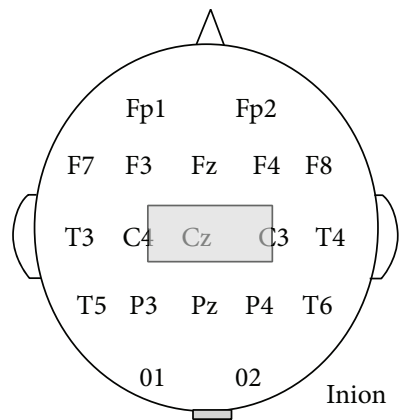

(b)

FIGURE 1: (a) A novel bihemispheric tDCS montage targeting right and left lower limb motor cortices simultaneously. (b) Illustrative tDCS montage using the international 10-20 EEG electrode placement. The anodal (stimulating) electrode was placed over Cz and covered a region $10-20 \%$ anterior to $\mathrm{Cz}$ as measured from the midpoint of the electrode. The cathode was placed over the inion.

From a cortical perspective, human locomotion relies upon a distributed neural network including primary motor, premotor areas, and, importantly, white matter connections thereof [6]. Not surprisingly, changes in the cerebral white matter, associated with vascular risk factors and frequently detected on imaging in the elderly [7], are associated with gait and balance dysfunction $[8,9]$. Clinically, these patients have a low gait velocity due to reduced stride length, long double support time, and broad based gait [10]. Small vessel disease (SVD) is an increasing cause of falls in the ageing population for which, critically, there is currently no treatment. The relevance of the primary motor and premotor cortices in the control of gait in relation to SVD has been demonstrated using fMRI [11] and cerebral perfusion techniques $[12,13]$. Given the lack of treatment options for this growing problem, we attempted to improve gait and balance function in these patients using noninvasive electrical stimulation that as we found can alter gait learning in healthy subjects [14].

\section{Current Treatments for Gait Disturbance}

Neurological gait and balance impairment has proved notoriously difficult to treat. For example, although some evidence suggests that freezing of gait in patients with Parkinson's disease may improve with levodopa [15], one study indicated that gait may worsen in medicated patients, perhaps as a result of drug-induced dyskinesia [16]. As a result, there has been a drive towards nonpharmacological therapies. Deep brain stimulation (DBS) has revolutionized the management of dopaminergic motor features in many patients with severe Parkinson's disease, and whilst there is some evidence in support of DBS in the treatment of gait disorders in these patients [17], its effect on the nondopaminergic motor features of Parkinson's disease, such as gait and balance, has been variable. Thus, one study showed that approximately $50 \%$ of freezers convert to nonfreezers after subthalamic nucleus DBS [18], although freezing of gait has also been shown to worsen after bilateral DBS [19]. In patients with resistant gait disturbance stimulation of the substantia nigra pars reticulata appears to improve axial motor features [20], including freezing of gait [21]. More recently DBS of the pedunculopontine nucleus (PPN) has shown variable outcomes on gait and balance [22-25].

Noninvasive treatment options, in particular repetitive transcranial magnetic stimulation, showed early promise in the treatment of gait in this group of patients [26, 27], but subsequent studies using intermittent magnetic stimulation [28] and tDCS [29] have yielded negative results. Physical therapy remains the mainstay of treatment for patients with neurological gait and balance dysfunction $[30,31]$ and is known to induce long-lasting plastic changes in the cerebral cortex [32]. We addressed an important but as yet unanswered question of whether the beneficial effects of physical training can be enhanced using noninvasive brain stimulation techniques. To do this we applied tDCS during physical training.

\section{Transcranial Direct Current Stimulation}

tDCS is a noninvasive neurostimulation technique that consists of delivering a weak current via the use of saline-soaked electrodes applied over the scalp. This has been shown to induce bidirectional polarity-dependent changes in cortical excitability of the underlying cortex. In general terms, anodal tDCS increases cortical excitability, and cathodal tDCS decreases it [33]. The physiological and behavioural effects of tDCS have been shown to last for up to one hour, implying that tDCS also modulates the synaptic microenvironment, modulating the synaptic strength of NMDA receptors, altering GABA-ergic activity, and modulating intracortical and corticospinal neurons [33-35].

\section{Bihemispheric tDCS for Gait}

Most studies using tDCS in the field of locomotor control have focused on stimulation over the lower limb primary 
motor cortex (M1) of a single hemisphere. So-called bihemispheric tDCS, however, aims to deliver cathodal stimulation to one motor cortex and anodal tDCS to the opposite hemisphere in an attempt to restore the interhemispheric imbalance caused by discrete cortical lesions (e.g., stroke) and to thus improve walking through its effects on the function of the hemiparetic limb.

Although there is some evidence that DBS reprogramming techniques such as "better side reduction" can reduce lower limb akinesia asymmetry and thus improve gait freezing [36], the cortical influences over locomotion are not lateralized. It is perhaps then surprising that there have not been attempts at applying electrical stimulation to both motor cortices simultaneously, particularly for nonstroke neurological gait disorders. We therefore attempted to modulate gait by applying tDCS to the primary motor and premotor cortices of both hemispheres simultaneously using a large midline electrode (Figure 1). Whilst the relative lack of focus of tDCS is an apparent disadvantage for physiological experiments, this may be welcomed when attempting to modulate complex and distributed cortical networks such as those involved in gait.

\section{Can tDCS Modulate Locomotor Activity in Healthy Individuals?}

Before tDCS can be considered as a therapeutic tool to treat patients with gait disorder, its effect upon lower limb excitability in healthy individuals needs to be evaluated. We posed two associated questions: first, can anodal tDCS over M1 and premotor cortex alter locomotor adaptation? Second, are the putative behavioural changes associated with tDCS related to neurophysiological changes in M1 cortical excitability of the legs?

To address the first question, we explored the effect of anodal tDCS over primary motor and premotor leg cortices on a locomotor adaptation task: the "broken escalator" paradigm - a laboratory reconstruction of the unusual sensation and physical stumble many people experience when stepping onto an escalator that is out-of-order [37-41]. This paradigm induces a locomotor aftereffect (forward trunk displacement and increased gait velocity) that reflects motor learning. We predicted that increasing the excitability of the primary motor and premotor cortical leg areas using anodal tDCS would increase the amplitude of the locomotor aftereffect. We further hypothesized that anodal tDCS over M1 would prolong the aftereffect given the role of $\mathrm{M} 1$ in memory retention. To address the second question we used TMS in a separate group of subjects to probe changes in cortical leg excitability before and after tDCS to M1.

For the gait experiments, we recruited 30 healthy participants that were allocated to 2 separate groups for testing. The "real tDCS" group received tDCS at $2 \mathrm{~mA}$ over 15 minutes, and "sham tDCS" group received sham stimulation for 15 minutes. Both groups performed 5 "BEFORE" trials by stepping onto a stationary sled, 5 MOVING (learning) trials stepping onto a moving platform, and 5 AFTER trials. The aftereffect is typically seen only in the 1st AFTER trial.
We applied tDCS to these subjects using a novel electrode montage that was determined using evidence from MRI studies [42] and three-dimensional probabilistic anatomic correlation techniques [43] showing that the scalp topography of $\mathrm{Cz}$ (international 10-20 EEG system [44]) corresponds to lower limb primary motor cortex. Thus a DC stimulating rectangular saline-soaked sponge electrode $(10 \times 4 \mathrm{~cm}$; surface area $40 \mathrm{~cm}^{2}$ ) was placed centrally across the scalp to cover a region $10-20 \%$ anterior to $\mathrm{Cz}$ as measured from the midline of the stimulating electrode (Figure 1). The reference electrode $(4 \times 4 \mathrm{~cm})$ was positioned at the inion. A $2-\mathrm{mA}$ current was delivered by a battery-driven Magstim Eldith DC stimulator (neuroConn, Ilmenau, Germany) between the end of the BEFORE trials and the beginning of the MOVING trials (i.e., just before the adaptation period). The current was initially increased by a ramp input over $10 \mathrm{~s}$ until reaching $2 \mathrm{~mA}$ (current density $0.05 \mathrm{~mA} / \mathrm{cm}^{2}$ ). Stimulation duration of $15 \mathrm{~min}$, as chosen, can result in an excitability change lasting up to $90 \mathrm{~min}$ [33]. The sham stimulation used for control purposes and is identical to the real stimulation condition except that the current drops off to zero after $30 \mathrm{~s}$.

We recorded forward trunk displacement and gait approach velocity using a position tracking device (Fastrak) attached to the subjects' back at C7 and electromyograms of the anterior tibialis and gastrocnemius muscles.

How can one be sure that tDCS as used here was having an effect on lower limb excitability? In a separate series of experiments we used transcranial magnetic stimulation that delivers a magnetic pulse to depolarise the cortical membrane over leg M1 and evaluating the effect of tDCS on TMS-induced motor-evoked potentials (MEPs) and silent period durations in tibialis anterior bilaterally in 16 additional healthy subjects. Magnetic stimuli were delivered to the motor cortex using an angled double-cone coil positioned over the tibialis anterior hotspot. TMS-induced MEPs from the tibialis anterior were recorded before and after tDCS (real stimulation in 8 subjects, sham in 8).

Finally, in a separate series of experiments we assess what effect, if any, cathodal stimulation over the inion (Figure 1) could have had on cerebellar structures, given the recognized role of the cerebellum in motor learning and adaptation [45]. We predicted that our electrode montage would spare cerebellar structures, as this is a suboptimal placement to stimulate the cerebellar cortex [46]. Eyeblink classic conditioning $(\mathrm{EBC})$ is a well-characterized experimental paradigm that is conserved across species and is dependent on the cerebellum [47] and consists of pairing a weak conditioning stimulus with a strong unconditioning stimulus repeatedly to produce conditioned responses consisting of an eyeblink starting before the US. EBC was evaluated in 8 subjects after receiving $\mathrm{tDCS}$ as applied in the main gait experiment.

The main finding was that the real tDCS group, compared with sham, displayed a $80 \%$ larger trunk sway $(P=0.04)$ and increased gait velocity (although not statistically significant; $P=0.15$ ) in the first AFTER trial (aftereffect) and a persistence of the trunk sway aftereffect into the second AFTER trial $(P=0.0013)$. Neither gait velocity nor trunk sway was 


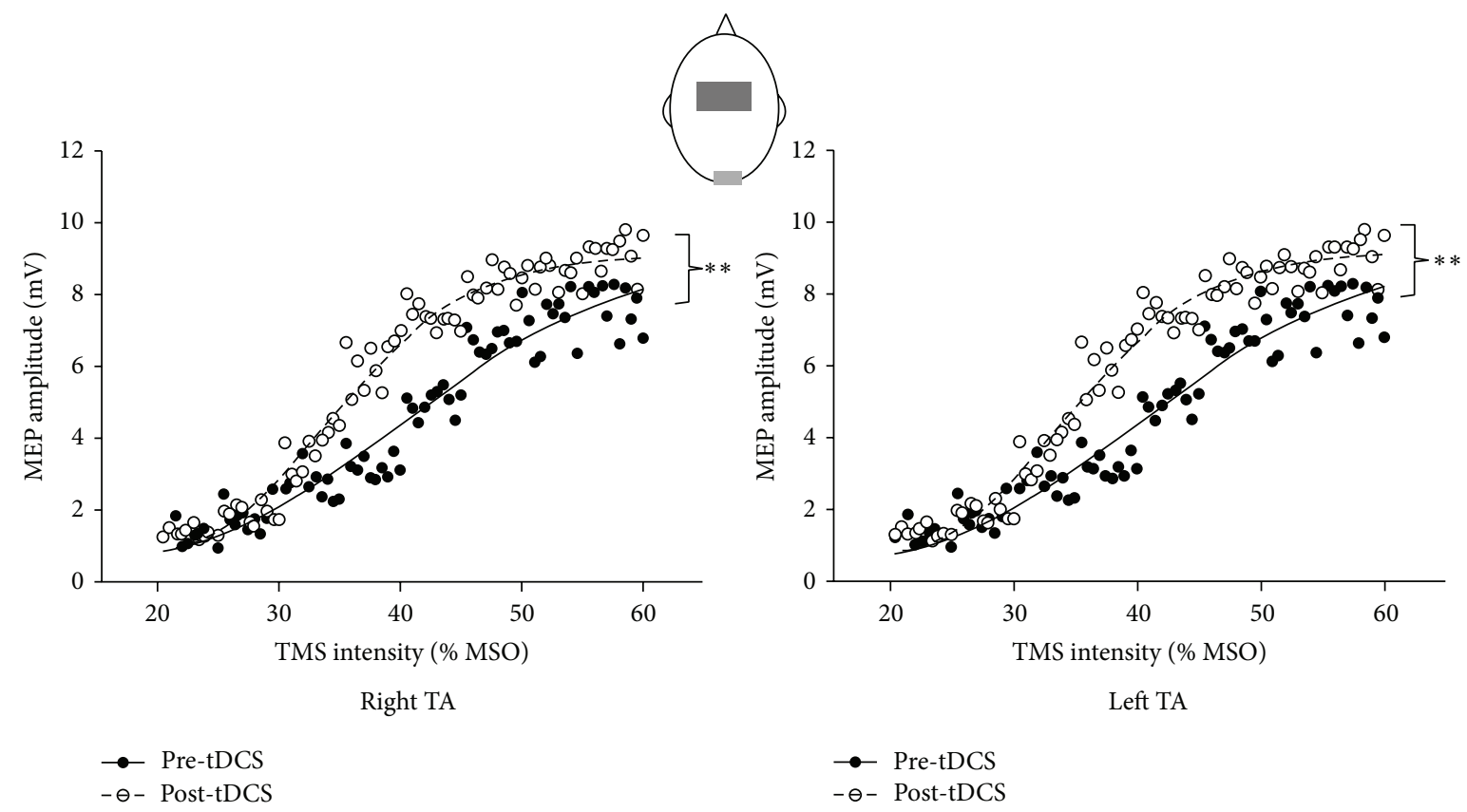

FIGURE 2: TMS-induced motor-evoked potential (MEP) amplitudes in right and left tibialis anterior (TA) muscles before and after tDCS or sham. Top: MEP amplitudes before (black spots) and after (white spots) real tDCS. Bottom: MEP amplitudes before and after sham stimulation. MSO, maximum stimulator output.

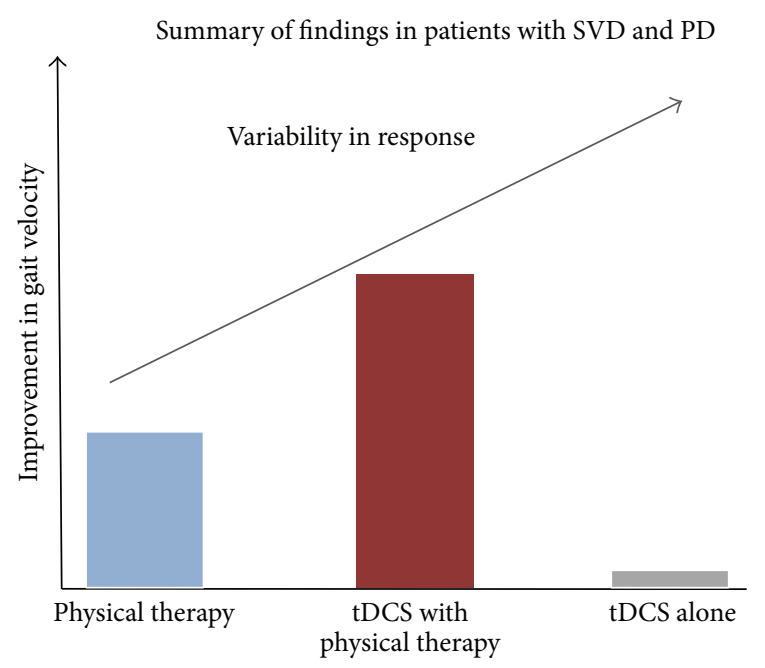

Figure 3: Summary of findings. The combination of tDCS with physical therapy resulted in the greatest improvements in gait velocity in patients with Parkinson's disease and small vessel disease; physical therapy improved gait, but tDCS alone had no significant effect. Notably, the variability in response was greatest in patients receiving $\mathrm{tDCS}$, indicating that some patients improved more than others.

significantly different between stimulation groups during the learning trials $(P=0.67)$. The EMG signals were rectified and integrated over a $500 \mathrm{~ms}$ time window after foot-sled contact and normalized with respect to mean BEFORE (trials 3-5) values. In keeping with results from forward trunk sway, EMG activity was significantly raised in the left and right medial gastrocnemius (MG) muscles in the tDCS group in the aftereffect trial $(P<0.001$ for left MG and $P=0.03$ for right $M G)$. Note that the left $M G$ is the muscle involved in breaking the forward momentum during the aftereffect as it is the left leg that makes the first contact on the sled.

Our TMS data revealed a significant increase in MEP amplitudes in right $(P<0.001)$ and left TA muscles $(P<0.001)$ following $15 \mathrm{~min}$ of $2 \mathrm{~mA}$ anodal tDCS using an inion reference electrode (as used in the broken escalator experiment) but not for sham stimulation (Figure 2). Concordantly, silent-period durations increased with real stimulation but not with sham.

Cathodal effects over the cerebellum would be expected to impair conditioned eyeblink responses. All subjects in the real tDCS group, however, and 7 subjects in the sham group $(n=8)$ acquired the conditioned response, indicating that placing the cathode electrode over the inion does not appear to have significant cerebellar effects.

In summary, we showed that stimulation of M1 and premotor cortex before skill acquisition increases the trunk overshoot component of the locomotor aftereffect, thus modulating locomotor adaptation. When the brain encounters environmental or sensory changes in the body, it must choose the most appropriate response for the current situation, using a Bayesian motor decision-making process [48]. Such a process of "risk assessment" may be subject to interactions from the physical properties of the sled and the individual's state of arousal. tDCS as used in this study may thus prevent the selection of a motor program that is contextually appropriate in favor of a cautious approach "the sled may move after all", thus generating a larger aftereffect. Alternatively, anodal tDCS may have increased neuronal excitability in a widespread cortical network with reinforcement of synaptic 
changes appropriate for stepping onto a moving sled as a result of the repetitive locomotor task. This in turn could lead to an enhanced expression of the adaptation aftereffect when stepping onto the stationary platform.

An interesting observation is that no significance was found for the gait velocity component of the locomotor aftereffect following tDCS. Data from previous work exploring the relationship between gait velocity and trunk overshoot components of the locomotor aftereffect have shown that they are dissociable [49]. Different neural mechanisms may underlie the trunk overshoot and gait velocity components of the aftereffect and may thus be subject to differential effects from tDCS although such a hypothesis warrants further exploration.

Our results could have clinical implications. The finding of an effect of cortical modulation on involuntary locomotor control is of potential interest for patients with gait disorders as tDCS offers the advantage of ease of access. Whilst there is evidence supporting the use of tDCS in upper limb rehabilitation following stroke [50] and Parkinson's disease [29], this is the first study to assess specifically the effect of bihemispheric anodal tDCS on gait. Results from the current finding suggest that direct noninvasive stimulation of primary motor cortex and premotor areas may be suitable sites to target locomotor adaptive learning [14]. Accordingly, our findings in healthy subjects support the use of tDCS for experimental treatment of neurological gait disorders.

\section{The Use of tDCS in Patients with Parkinson's Disease}

Based on our findings that tDCS was able to increase excitability in lower limb motor cortex and increase locomotor adaptation, we next applied anodal tDCS using the same bihemispheric tDCS montage in patients with Parkinson's disease (PD). PD is a common cause of gait and postural instability but it is generally accepted that these features are largely levodopa resistant $[3,51-54]$. An early study assessing levodopa responsiveness for various gait parameters identified that temporal parameters such as stride and swing duration and stride duration variability were levodopa resistant, whereas stride length was levodopa sensitive [55]. More recent evidence suggests that levodopa may differentially improve proximal more than distal lower limb kinematics [56]. Interestingly, levodopa appears to have no effect on locomotor adaptation [57].

Given the reduced activity in premotor and primary motor cortical regions in Parkinson's disease [58], these areas may be important therapeutic noninvasive neurostimulatory targets for patients with Parkinson's disease and gait disturbance [59].

It is recognized that physical therapy induces physiological changes in the primary motor cortex of patients with Parkinson's disease [32]. tDCS has also been shown to improve motor learning and rehabilitation in PD [60], although outcomes have been mixed [61]. Previous studies using noninvasive brain stimulation in patients with PD did not find reproducible benefits on gait [28, 29], although stimulation paradigms were not combined with physical therapy.

We hypothesised that anodal tDCS time locked to physical training would improve gait and balance in Parkinson's disease above and beyond the effects of tDCS in isolation. Our primary gait outcome was gait velocity as this is a functional marker of disease severity, and our primary balance outcome was recovery of stance in the pull test [62], with postural instability being a common cause of falls in these patients.

Sixteen patients were recruited (average age $76.4,(\mathrm{SD}=$ 5.7); disease duration 10.4 yrs (3.0); UPDRS III 25.8 (5.7)). Patients were assigned to one of two groups; Group I "physical-training" $(n=8)$ and Group II "no physicaltraining" $(n=8)$. Patients in Group I received tDCS during a fifteen-minute period of physical training and those in Group II received only tDCS, without physical training. For both groups, the application of tDCS was also randomised to either real tDCS stimulation or sham stimulation in the first session. All subjects returned 1 week later, to allow an appropriate wash-out period for the neurostimulation. In this second session subjects in the "physical training" group again received physical therapy, but those that had received real stimulation in the first session were given sham stimulation, and vice versa. Patients in the "No physical training" group again received only neurostimulation in this second session, with those that received real stimulation in the first session given sham stimulation, and vice versa. Outcomes were recorded prior to any intervention (baseline) and after intervention (final assessment). The physical therapy protocol lasted 15 minutes with a focus on improving gait initiation, stride length, gait velocity, arm swing, and balance.

Statistical analysis consisted of paired student $t$-tests, with appropriate Bonferroni correction. To assess the effect of combining tDCS with physical training we compared the average \% change in performance in patients receiving real stimulation in Group I versus real stimulation in Group II. We compared the average \% change in performance in patients receiving real versus sham stimulation in Group II to assess the isolated effect of tDCS stimulation (independent of physical training). Lastly, to assess the isolated effect of physical training (independent of tDCS) we compared the average $\%$ change in performance in patients receiving sham in Group I versus patients receiving sham stimulation in Group II. Where an isolated effect of physical training was observed, we compared the average $\%$ change in performance in patients receiving real stimulation in Group I versus sham stimulation in Group I, to assess the effect of combining tDCS with physical training - above and beyond the effect of physical training alone.

We used an identical tDCS montage and stimulation protocol as described in the initial healthy subject study above. The current was delivered for 15 minutes during training ("physical training" group) or during the execution of the gait and balance tasks ("No physical training" group). Electrodes were secured onto the scalp during the assessments using a modified diving head cap and tDCS battery fitted on a lightweight backpack. 
The primary outcome for gait was gait velocity. Secondary outcomes included stride length, $3 \mathrm{~m}$ "Timed Up and Go," $6 \mathrm{~m}$ walk. The primary balance outcome was a quantitative pull test that is a clinical tool used to evaluate postural instability in patients with Parkinson's disease [63].

We found that the combination of anodal tDCS with concurrent physical therapy increased gait velocity $(P<$ $0.001)$, stride length $(P=0.01)$, the time taken to complete the "Timed Up and Go" $(P=0.04)$, and the $6 \mathrm{~m}$ walk $(P=0.04)$. Whilst there were modest benefits in patients receiving only physical therapy, these were comparatively less than when therapy was combined with tDCS. We did not observe any benefits of isolated tDCS (i.e., without physical therapy) [64].

For balance, the combination of tDCS and physical therapy reduced the time taken to regain stability following the retropulsion stimulus compared to tDCS alone $(P=0.01)$. Again, there was no isolated effect of stimulation $(P=0.94)$ or physical therapy $(P=0.87)$.

In summary, we found a significant benefit of combining tDCS with physical training for gait velocity (primary gait outcome) and the performance on the pull test (primary balance outcome), but there was no isolated effect for tDCS or physical training [64].

Physical training in Parkinson's disease normalises cortical excitability in M1 [32], whilst tDCS may lower the threshold for these changes to occur (Figure 5). Thus, combining physical gait and balance therapy with tDCS over primary motor and premotor cortex has a physiological basis.

One interesting finding from this study was that patients with more severe motor symptoms (UPDRS III and Hoehn Yahr scale) tended to show a greater improvement in walking time with tDCS compared to patients with milder disease [64]. Although the number of patients tested is insufficient to draw firm conclusions from a correlation analysis, our data suggest that patients with more advanced disease are more likely to benefit from combined DC stimulation and physical therapy than patients with mild disease. In contrast, individual gait outcomes did not correlate with age, the degree of leukoaraiosis, or cognitive impairment, suggesting that individual patients at the extremes of the disease process may be less likely to respond to neurostimulation.

Whilst our patient sample size lacks the power to draw conclusive results, our preliminary data suggests that the tDCS may be a useful adjunct to physical therapy to improve gait in patients with Parkinson's disease. Unfortunately, the lack of follow-up assessments meant that we were unable to comment on possible longer-term benefit of the interventions, or indeed whether repeated tDCS sessions lead to greater and sustained improvements.

\section{Combining tDCS and Dance Therapy in a Single Patient with Parkinson's Disease}

A hallmark of progression of PD is postural instability and associated falls [65]. Dance and movement therapy has been shown to improve balance and gait in patients with gait disturbances [66], with the Argentine tango receiving particular interest in patients with PD [67]. There is evidence that tango dancing may alter cortical excitability, with increased activity over supplementary motor and premotor cortices in healthy subjects during imagined walking following a tango lesson [68]. Given our findings that tDCS improves gait in patients with PD, we decided to explore the effect of applying anodal tDCS over primary motor and premotor cortex on trunk kinematics in a patient with moderate PD during tango dancing.

We applied transcranial direct current stimulation (tDCS) in a 79-year-old male patient with moderate PD (UPDRS III = 34, HY scale $=$ stage 3 ; disease duration 7 yrs) during tango dancing to assess its effect on trunk motion and balance [69]. The patient performed a total of four dances ("The butterfly," Osvaldo Pugliese orquestra, music by Pedro Maffia, and lyrics by Celedonio Flores) over two days; two "tango and tDCS" and two "tango and sham" in a randomised double-blind fashion.

We recorded the average sagittal (pitch) and coronal (roll) trunk peak-to-peak velocity was measured across the whole dance using digitally based angular-velocity transducers attached to the patients lower back [70]. We also recorded a Tinetti Gait index questionnaire [71] (which is performed on a three-point ordinal scale assessing task-oriented gait and balance parameters, such as the ability to rise from a chair, standing balance, step symmetry, etc.) and obtained a subjective measure of performance before and after each dance session from the patient's professional dance partner, who was blinded to the intervention.

As in the studies reported above, a DC stimulating rectangular saline-soaked sponge electrode $(10 \mathrm{~cm} \times 4 \mathrm{~cm})$ was placed centrally across the scalp to cover a region $10-$ $20 \%$ anterior to $\mathrm{Cz}$ as measured from the midline of the stimulating electrode. The reference electrode $(4 \mathrm{~cm} \times 4 \mathrm{~cm})$ was positioned at the inion (Figure 1). A $2 \mathrm{~mA}$ current was delivered by a battery-driven Magstim Eldith DC stimulator (NeuroConn, Germany) during the Tango dance. The current was initially increased by a ramp input over $10 \mathrm{~s}$ until reaching $2 \mathrm{~mA}$ (current density $0.05 \mathrm{~mA} / \mathrm{cm}^{2}$ ).

We found that trunk peak velocity during tango was significantly greater during tDCS compared to sham stimulation [69] (Figure 4(a)). The questionnaire data revealed subjective improvements in dance performance, and the dance partner commented that the patient performed an unusually greater number of choreographed steps in one of the sessions, which was later found to correspond to a tDCS (rather than sham) session. Improvements were also seen in the semiquantitative gait function assessment (Tinetti Gait Index: $t$-test; $P=0.04$ ).

Our findings suggest that $\mathrm{tDCS}$ may be a useful adjunct to gait rehabilitation for patients with $\mathrm{PD}$, although studies in a larger group of patients are needed to evaluate the therapeutic use of noninvasive brain stimulation during dance therapy.

\section{The Use of tDCS in Patients with Small Vessel Disease with Gait Disturbance}

Whilst physical therapy has been used in conjunction with tDCS to augment the effects of gait training [72] and lower 


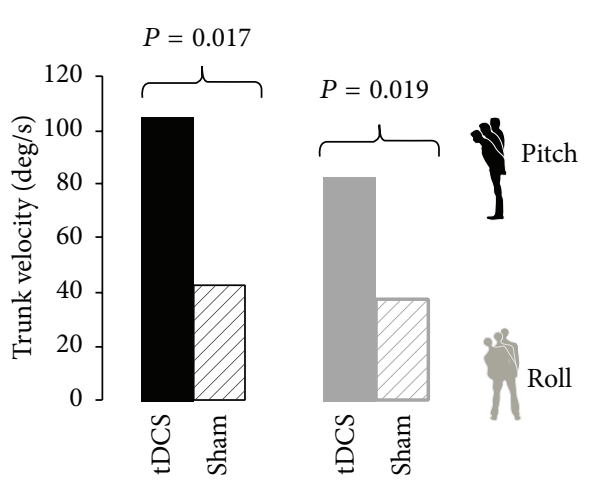

(a)

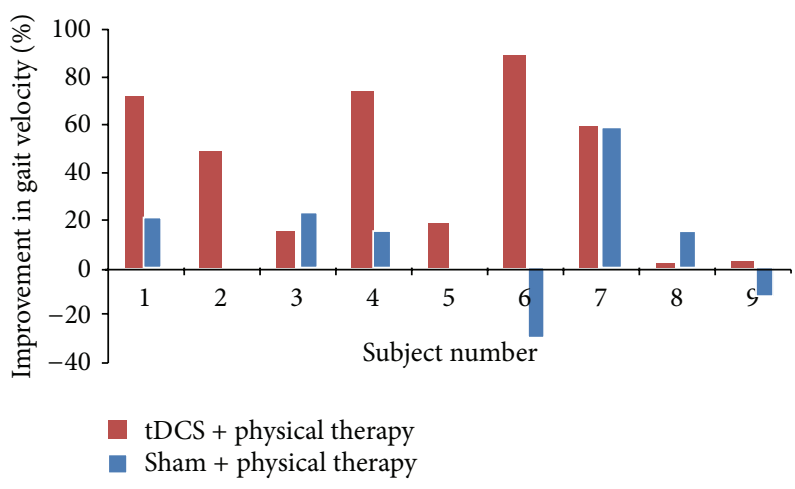

(b)

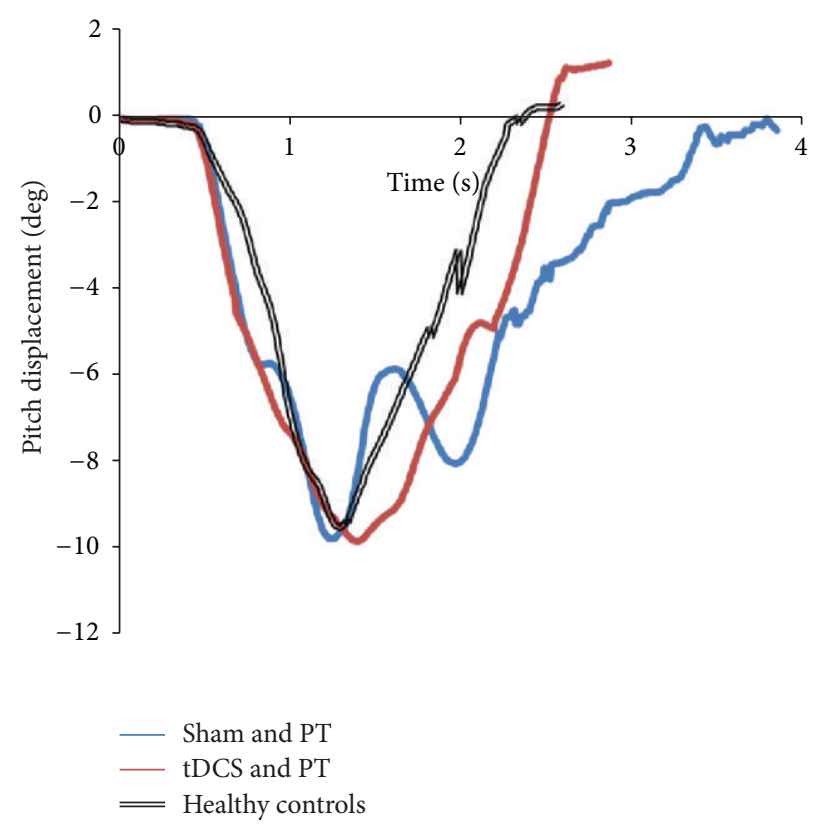

(c)

FIgURE 4: (a) Averaged data for the 4 tango dances showing increased trunk velocity in coronal (roll) and sagittal (pitch) planes in the real tDCS sessions, compared to sham. (b) Individual data from patients with small vessel disease showing percentage change in gait velocity following real tDCS (red) and sham stimulation (blue). (c) Time taken to regain a pitch angular displacement of $+/-2 \mathrm{~cm}$ of baseline following a backward pull on the shoulders for tDCS with physical therapy, sham with physical therapy in patients with Parkinson's disease, and healthy aged-matched controls.

limb rehabilitation [73] in patients with hemiparesis following established cortical strokes, the combination of noninvasive stimulation with simultaneous physical therapy has not been previously studied in patients with primary gait disorders such as small vessel disease.

Small vessel disease (white matter lesions or leukoaraiosis) describes hyperintensities in the cerebral subcortical white matter and is associated with gait and balance dysfunction and falls in the elderly [8] for which there are no evidence-based treatments.

Primary motor and premotor cortices are relevant to the control of gait in relation to small vessel disease [11, 12]. We attempted to facilitate neural activity in this network by combining physical training with $\mathrm{tDCS}$ of the primary motor leg area and premotor cortex bilaterally [14]. We recruited
9 patients with SVD (mean age 79.4 years, SD $=5.5$; mean minimental state examination score 27.0; Fazekas score 2.4).

The primary gait outcomes were gait velocity and stride length, which correlate with disease severity in these patients [74]. The primary balance outcome was recovery of stance in the retropulsion test, with postural instability being a feature of small vessel disease and a common cause of falls in these patients [75]. Secondary outcomes were timed walk (a surrogate measure of gait velocity) and angular trunk velocity and amplitude.

Subjects performed a $6 \mathrm{~m}$ walk, Timed Up and Go (TUG), and retropulsion test twice in each session: "baseline" and "final" assessments. Following "baseline" assessments patients underwent a 15-minute balance and gait training session concurrently with anodal tDCS stimulation (real or 


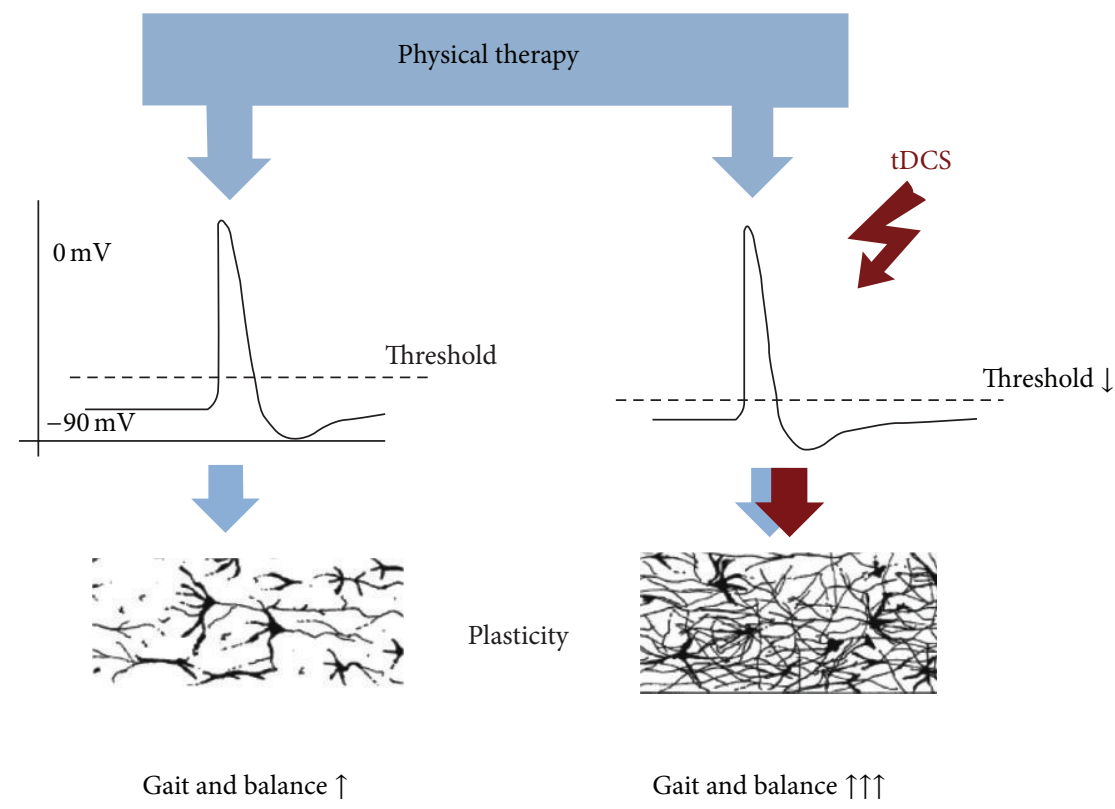

FIGURE 5: Proposed neural mechanism for combining tDCS with physical therapy. Physical therapy induces cortical changes that are thought to underlie the functional improvement in motor function. tDCS alters cortical excitability, perhaps reducing the threshold for these changes to occur when combined with physical therapy, resulting in greater cortical plasticity and better motor outcomes than physical therapy alone.

sham). Subjects then immediately repeated the gait and balance tests ("final" assessment). Assessments took on average approximately 15 minutes to complete.

A device encompassing two digitally based angularvelocity transducers arranged to measure angular trunk displacement and velocity in the roll (coronal) and pitch (sagittal) planes (SwayStar System, Balance Int. Innovations $\mathrm{GmbH}$, Switzerland) was used in all subjects. The transducers were encased in a lightweight Bluetooth cordless device attached to an elasticised motorcycle belt, which was easily fitted on the subjects back at the L2-3 level. Angular deviations were calculated using online-trapezoid integration of the angular velocities [70].

Anodal tDCS was applied to the motor cortex of both hemispheres simultaneously using the same electrode montage and protocol previously described (Figure 1). The current was delivered during the exercise session for 15 minutes. Sham stimulation was identical to real stimulation except that the current was delivered for only 30 seconds and then switched off [76]. As part of a proof-of-principle study each patient received a single session of active (and sham) tDCS rather than repeated treatment sessions.

The principal result was that the combination of tDCS and locomotor training improves clinically relevant locomotor performance as measured by stride length $(P=0.047)$, stride length variability $(P=0.001)$, and gait velocity $(P=$ 0.008; Figure 4(b)) in patients with leukoaraiosis [77]. We also observed improvements in the time taken to regain posture following a retropulsive stimulus in the tDCS and physical therapy group, but not with tDCS or physical therapy alone $(P=0.045$; Figure $4(\mathrm{c}))$.
Our results support the finding that tDCS applied during the motor practice ("voluntary drive" [78]) improves performance $[73,79]$. Although we cannot comment on whether this effect relates to motor practice or motor learning, our previous work described here has shown improvements in motor adaptive learning in healthy subjects using an identical tDCS montage [14].

This result suggests that targeting neocortical regions involved with gait using noninvasive stimulation may be one therapeutic strategy to overcome the corticosubcortical disconnection caused by white matter lesions in leukoaraiosis [77].

One explanation for our findings is that the voluntary motor activity (versus external involuntary robotic assistance, e.g.) occurring during physical training induces changes in excitability in relevant motor cortex [80]. Such neural activity strengthens corticospinal and intracortical networks, and tDCS may lower the threshold for these plastic changes to occur (Figure 5). Although the magnitude of the increase in gait velocity observed was somewhat unexpected, motor improvements of this magnitude have been previously observed within single sessions combining noninvasive brain stimulation techniques with peripheral nerve stimulation [81]. Further studies in a larger cohort of patients will help determine the everyday clinical applicability of this technique.

The effect of tDCS on balance in such patients has not been previously investigated. Whilst the response to the retropulsion test undoubtedly involves a coordinated neuronal network including cortical (M1) and subcortical (basal ganglia) structures and vestibular and spinal reflexes, 
one unanswered question was whether tDCS could impact on this network, either through direct actions on the motor cortex, or as a result of the more widespread effects of tDCS that are increasingly recognised [82]. Nevertheless, given that the retropulsion test is abnormal in patients with frontobasal ganglia disorders, the observed effects of tDCS most likely reflect changes to corticosubcortical networks. The combination of physical therapy and offline tDCS (applied during learning rather than execution of the task) improves balance in patients with leukoaraiosis that may be related to preferential effects on reflex activity, rather than voluntary motor performance [83]. Alternatively, neurostimulatory effects over phasic execution of movement such as a postural response may result from an increase in spontaneous neuronal firing rate [84]. Further studies dissecting the underlying mechanisms involved are warranted.

\section{The Use of tDCS in Patients with Gait Disturbance following Subacute Stroke}

A third of stroke survivors become unable to walk as a consequence of their stroke [85]. This functional deficit imposes a significant burden upon patients and carers and explains why gait recovery is a major focus of poststroke rehabilitation [86]. Despite the impact of gait impairment upon quality of life, most evidence that tDCS may boost the efficacy of conventional physical therapy has been obtained for poststroke upper limb motor recovery [87-89].

The application of tDCS to improve gait in patients with stroke necessarily differs from its application in patients with diffuse cortical or subcortical neurological disease. Thus, in bihemispheric tDCS the anode and cathode are placed over separate hemispheres [90] to simultaneously upregulate and downregulate activity in opposite hemispheres. Such tDCSinduced activity is theorised to temporarily restore the interhemispheric excitability balance that is disrupted following unilateral stroke [91]. A rebalancing of interhemispheric activity may be pivotal to promote functional recovery poststroke. Bihemispheric tDCS applied in this way enhances upper limb recovery in both chronic and subacute stroke patients [88]. Whether such effects extend to gait rehabilitation has not been explored. The subacute stroke phase represents a period of considerable neural plasticity [92] and thus a prime opportunity to influence such adaptive mechanisms using noninvasive brain stimulation.

Fourteen patients with subacute hemispheric stroke (2-8 weeks aftere stroke) were randomised to either active ( $n=$ 7 ; mean age 67.5 years $(\mathrm{SD}=11.8)$; modified Rankin scale $2.1(\mathrm{SD}=1.1))$ or sham $(n=7 ; 56.4$ years $(\mathrm{SD}=12.4)$; modified Rankin scale 2.6 $(\mathrm{SD}=1.1))$ tDCS. The anode was placed over the ipsilesional lower limb primary motor cortex and the cathode over the contralesional leg motor cortex. A blinded assessor measured performance in the Timed Up and Go (TUG) test and the Performance Oriented Mobility Assessment (POMA; a 0-28 point ordinal scale where 28 is indicative of independent mobility and balance, with a low falls risk and 0 is most impaired). Assessments were performed before and after active tDCS or sham tDCS.

The average difference scores (i.e., posttest minus pretest) for the TUG test showed that, despite their older age, the active group was significantly faster following tDCS by $4.64 \mathrm{~s}$ whereas the sham group improved by only $0.01 \mathrm{~s}$ following sham stimulation ( $P=0.02$ for tDCS versus sham).

There was no significant difference between the two groups when comparing the difference in POMA scores after stimulation (POMA difference score for active group $=0.3$; POMA difference score for sham group $=0.3 ; P=0.90$; Mann-Whitney $U$-test). In the sham group, no participant improved by a clinically meaningful amount; however, one subject in the active tDCS group did improve by a clinically meaningful level (4 points).

We found that active tDCS is safe and uniformly reduced walking time in patients with subacute stroke compared to sham. In contrast, the gait and balance subscale scores of the performance-orientated mobility assessment were not improved by $\mathrm{tDCS}$. The differential benefits of $\mathrm{tDCS}$ on gait speed versus no effect on the POMA score may reflect the fact that the POMA is an ordinal scale, whereas gait speed is a continuous measure and may thus be more sensitive to detect change.

Notably, the two patients showing greatest improvements with tDCS also had comparatively worse baseline scores for the TUG, suggesting that the effects of tDCS may be more pronounced in more severely affected patients. One subject from the sham stimulation group showed a nearly 4-second improvement in the time to complete the TUG. This may represent a large placebo effect in a patient who may not have felt confident to walk to his maximum capability before the stimulation. Alternatively, the effect size may reflect the nature of the subcortical stroke-gait function in patients with subcortical white matter disease can be more variable [74], perhaps relating to fluctuating attentional drive directed towards walking.

Overall, we have shown that a single session of anodal bihemispheric tDCS can improve gait speed in patients with subacute stroke [93]. Additional larger studies will be required to see if these benefits can be replicated and sustained with repeated treatment sessions and if there is indeed a differential response to tDCS between subcortical versus cortical stroke.

\section{Summary of Findings and Future Work}

There are surprisingly few studies addressing the use of noninvasive cortical stimulation for the treatment of neurological gait disorders. Repetitive transcranial magnetic stimulation, for example, showed early promise in the treatment of gait in patients with Parkinson's disease [26, 27], but subsequent studies using intermittent magnetic stimulation [28] and tDCS [29] have yielded negative results. One study assessed the effect of tDCS when applied concomitantly with gait rehabilitation in patients with chronic stroke finding no additional benefit over and above rehabilitation alone [72]. 
Studies assessing the effect of tDCS in upper limb rehabilitation variously report benefit in both chronic and subacute strokes [87, 88]. In contrast, one large study [94] found no improvement in clinical outcome with acute (i.e., 48 hours) tDCS.

Firstly, this work has shown that it is possible to target lower limb muscles cortically, and that this can be done for both legs simultaneously by placing a central large anodal electrode over the motor strip and a smaller cathode electrode over the inion. Using a combination of TMS with tDCS it has been possible to show that such a montage increases cortical excitability of lower limb muscles of both legs.

Secondly, such cortical stimulation can be combined with physical therapy to enhance the effects of physical activity alone, for patients with Parkinson's disease and with small vessel disease and gait disturbance-an increasingly common cause of falls in the elderly.

Whilst the findings of these studies suggest that noninvasive electrical stimulation techniques may be a useful adjunct to physical therapy in patients with neurological gait disorders, there remains a need for further large mutlicentre randomized sham-controlled studies to evaluate the clinical relevance of such therapies. One striking feature across these and many other studies using noninvasive electrical stimulation is the intra- and interindividual variability in the behavioural responses following stimulation (Figure 3). One problem is that tDCS-related behavioural outcome measures are presumably dependent on factors outside the stimulation, such as motivation and attention that are difficult to control experimentally. As such, large numbers of patients and perhaps a range of electrode montages with computational modeling and direct imaging of current flow will be required to enable a translation of tDCS from experimental use into mainstream clinical practice for the treatment of gait disorders.

\section{Conflict of Interests}

The authors declare that there is no conflict of interests regarding the publication of this paper.

\section{References}

[1] J. G. Nutt, C. D. Marsden, and P. D. Thompson, "Human walking and higher-level gait disorders, particularly in the elderly," Neurology, vol. 43, no. 2, pp. 268-279, 1993.

[2] J. G. Nutt, "Classification of gait and balance disorders," Advances in Neurology, vol. 87, pp. 135-141, 2001.

[3] M. A. Hely, J. G. L. Morris, W. G. J. Reid, and R. Trafficante, "Sydney Multicenter Study of Parkinson's disease: non-L-doparesponsive problems dominate at 15 years," Movement Disorders, vol. 20, no. 2, pp. 190-199, 2005.

[4] H. Fukuyama, Y. Ouchi, S. Matsuzaki et al., "Brain functional activity during gait in normal subjects: a SPECT study," Neuroscience Letters, vol. 228, no. 3, pp. 183-186, 1997.

[5] I. Miyai, H. C. Tanabe, I. Sase et al., "Cortical mapping of gait in humans: a near-infrared spectroscopic topography study," NeuroImage, vol. 14, no. 5, pp. 1186-1192, 2001.
[6] G. E. Alexander and M. D. Crutcher, "Preparation for movement: neural representations of intended direction in three motor areas of the monkey," Journal of Neurophysiology, vol. 64, no. 1, pp. 133-150, 1990.

[7] O. Franch, L. Calandre, J. Alvarez-Linera, E. D. Louis, F. Bermejo-Pareja, and J. Benito-Leon, "Gait disorders of unknown cause in the elderly: clinical and MRI findings," Journal of the Neurological Sciences, vol. 280, no. 1-2, pp. 84-86, 2009.

[8] C. Blahak, H. Baezner, L. Pantoni et al., "Deep frontal and periventricular age related white matter changes but not basal ganglia and infratentorial hyperintensities are associated with falls: cross sectional results from the LADIS study," Journal of Neurology, Neurosurgery and Psychiatry, vol. 80, no. 6, pp. 608613, 2009.

[9] R. A. Bhadelia, L. L. Price, K. L. Tedesco et al., "Diffusion tensor imaging, white matter lesions, the corpus callosum, and gait in the elderly," Stroke, vol. 40, no. 12, pp. 3816-3820, 2009.

[10] K. Iseki, T. Hanakawa, K. Hashikawa et al., "Gait disturbance associated with white matter changes: a gait analysis and blood flow study," NeuroImage, vol. 49, no. 2, pp. 1659-1666, 2010.

[11] P. Linortner, F. Fazekas, R. Schmidt et al., "White matter hyperintensities alter functional organization of the motor system," Neurobiology of Aging, vol. 33, no. 1, pp. 197.el-197.e9, 2012.

[12] P. Linortner, F. Fazekas, R. Schmidt et al., "White matter hyperintensities alter functional organization of the motor system," Neurobiology of Aging, vol. 33, no. 1, pp. 197-e9, 2012.

[13] H.-J. Freund and H. Hummelsheim, "Lesions of premotor cortex in man," Brain, vol. 108, no. 3, pp. 697-733, 1985.

[14] D. Kaski, S. Quadir, M. Patel, N. Yousif, and A. M. Bronstein, "Enhanced locomotor adaptation aftereffect in the "broken escalator" phenomenon using anodal tDCS," Journal of Neurophysiology, vol. 107, no. 9, pp. 2493-2505, 2012.

[15] S. Fahn, "Levodopa and the progression of Parkinson's disease," The New England Journal of Medicine, vol. 351, no. 24, pp. 2498 2566, 2004.

[16] Q. J. Almeida, J. S. Frank, E. A. Roy, A. E. Patla, and M. S. Jog, "Dopaminergic modulation of timing control and variability in the gait of Parkinson's disease," Movement Disorders, vol. 22, no. 12, pp. 1735-1742, 2007.

[17] A. Stefani, A. Peppe, M. Pierantozzi et al., "Multi-target strategy for Parkinsonian patients: the role of deep brain stimulation in the centromedian-parafascicularis complex," Brain Research Bulletin, vol. 78, no. 2-3, pp. 113-118, 2009.

[18] S. Vercruysse, W. Vandenberghe, L. Münks, B. Nuttin, H. Devos, and A. Nieuwboer, "Effects of deep brain stimulation of the subthalamic nucleus on freezing of gait in Parkinson's disease: a prospective controlled study," Journal of Neurology, Neurosurgery and Psychiatry, vol. 85, no. 8, pp. 871-877, 2014.

[19] A. M. S. Muniz, J. Nadal, K. E. Lyons, R. Pahwa, and W. Liu, "Long-term evaluation of gait initiation in six Parkinson's disease patients with bilateral subthalamic stimulation," Gait and Posture, vol. 35, no. 3, pp. 452-457, 2012.

[20] N. Chastan, G. W. M. Westby, J. Yelnik et al., "Effects of nigral stimulation on locomotion and postural stability in patients with Parkinson's disease," Brain, vol. 132, no. 1, pp. 172-184, 2009.

[21] D. Weiss, M. Walach, C. Meisner et al., "Nigral stimulation for resistant axial motor impairment in Parkinson's disease? A randomized controlled trial," Brain, vol. 136, no. 7, pp. 20982108, 2013. 
[22] D. Nosko, M. U. Ferraye, V. Fraix et al., "Low-frequency versus high-frequency stimulation of the pedunculopontine nucleus area in Parkinson's disease: a randomised controlled trial," Journal of Neurology, Neurosurgery, and Psychiatry, 2014.

[23] M. U. Ferraye, B. Debû, V. Fraix et al., "Effects of pedunculopontine nucleus area stimulation on gait disorders in Parkinson's disease," Brain, vol. 133, part 1, pp. 205-214, 2010.

[24] P. Plaha and S. S. Gill, "Bilateral deep brain stimulation of the pedunculopontine nucleus for Parkinson's disease," NeuroReport, vol. 16, no. 17, pp. 1883-1887, 2005.

[25] E. Moro, C. Hamani, Y.-Y. Poon et al., "Unilateral pedunculopontine stimulation improves falls in Parkinson's disease," Brain, vol. 133, no. 1, pp. 215-224, 2010.

[26] E. M. Khedr, J. C. Rothwell, O. A. Shawky, M. A. Ahmed, and A. Hamdy, "Effect of daily repetitive transcranial magnetic stimulation on motor performance in Parkinson's disease," Movement Disorders, vol. 21, no. 12, pp. 2201-2205, 2006.

[27] M. P. Lomarev, S. Kanchana, W. Bara-Jimenez, M. Iyer, E. M. Wassermann, and M. Hallett, "Placebo-controlled study of rTMS for the treatment of Parkinson's disease," Movement Disorders, vol. 21, no. 3, pp. 325-331, 2006.

[28] D. H. Benninger, B. D. Berman, E. Houdayer et al., "Intermittent theta-burst transcranial magnetic stimulation for treatment of Parkinson disease," Neurology, vol. 76, no. 7, pp. 601-609, 2011.

[29] D. H. Benninger, M. Lomarev, G. Lopez et al., "Transcranial direct current stimulation for the treatment of Parkinson's disease," Journal of Neurology, Neurosurgery and Psychiatry, vol. 81, no. 10, pp. 1105-1111, 2010.

[30] I. Boers, W. Gerschlager, P. A. Stalenhoef, and B. R. Bloem, "Falls in the elderly II. Strategies for prevention," Wiener Klinische Wochenschrift, vol. 113, no. 11-12, pp. 398-407, 2001.

[31] K. H. Deane, D. Jones, E. D. Playford, Y. Ben-Shlomo, and C. E. Clarke, "Physiotherapy for patients with Parkinson's Disease: a comparison of techniques," The Cochrane Database of Systematic Reviews, no. 3, Article ID CD002817, 2001.

[32] B. E. Fisher, A. D. Wu, G. J. Salem et al., "The effect of exercise training in improving motor performance and corticomotor excitability in people with early Parkinson's disease," Archives of Physical Medicine and Rehabilitation, vol. 89, no. 7, pp. 12211229, 2008.

[33] M. A. Nitsche and W. Paulus, "Sustained excitability elevations induced by transcranial DC motor cortex stimulation in humans," Neurology, vol. 57, no. 10, pp. 1899-1901, 2001.

[34] D. Liebetanz, M. A. Nitsche, F. Tergau, and W. Paulus, "Pharmacological approach to the mechanisms of transcranial DCstimulation-induced after-effects of human motor cortex excitability," Brain, vol. 125, no. 10, pp. 2238-2247, 2002.

[35] M. A. Nitsche, L. G. Cohen, E. M. Wassermann et al., "Transcranial direct current stimulation: state of the art 2008," Brain Stimulation, vol. 1, no. 3, pp. 206-223, 2008.

[36] A. Fasano, J. Herzog, E. Seifert et al., "Modulation of gait coordination by subthalamic stimulation improves freezing of gait," Movement Disorders, vol. 26, no. 5, pp. 844-851, 2011.

[37] R. F. Reynolds and A. M. Bronstein, "The broken escalator phenomenon: aftereffect of walking onto a moving platform," Experimental Brain Research, vol. 151, no. 3, pp. 301-308, 2003.

[38] K. L. Bunday, R. F. Reynolds, D. Kaski, M. Rao, S. Salman, and A. M. Bronstein, "The effect of trial number on the emergence of the "broken escalator" locomotor aftereffect," Experimental Brain Research, vol. 174, no. 2, pp. 270-278, 2006.
[39] A. M. Bronstein, K. L. Bunday, and R. Reynolds, "What the "broken escalator" phenomenon teaches us about balance," Annals of the New York Academy of Sciences, vol. 1164, pp. 82-88, 2009.

[40] K. L. Bunday and A. M. Bronstein, "Locomotor adaptation and aftereffects in patients with reduced somatosensory input due to peripheral neuropathy," Journal of Neurophysiology, vol. 102, no. 6, pp. 3119-3128, 2009.

[41] D. A. Green, K. L. Bunday, J. Bowen, T. Carter, and A. M. Bronstein, "What does autonomic arousal tell us about locomotor learning?" Neuroscience, vol. 170, no. 1, pp. 42-53, 2010.

[42] T. D. Lagerlund, F. W. Sharbrough, C. R. Jack Jr. et al., "Determination of 10-20 system electrode locations using magnetic resonance image scanning with markers," Electroencephalography and Clinical Neurophysiology, vol. 86, no. 1, pp. 7-14, 1993.

[43] M. Okamoto, H. Dan, K. Sakamoto et al., “Three-dimensional probabilistic anatomical cranio-cerebral correlation via the international 10-20 system oriented for transcranial functional brain mapping," NeuroImage, vol. 21, no. 1, pp. 99-111, 2004.

[44] G. H. Klem, H. O. Lüders, H. H. Jasper, and C. Elger, "The ten-twenty electrode system of the International Federation. The International Federation of Clinical Neurophysiology," Electroencephalography and Clinical Neurophysiology, vol. 52, pp. 3-6, 1999.

[45] J. M. Galea, A. Vazquez, N. Pasricha, J.-J. O. de Xivry, and P. Celnik, "Dissociating the roles of the cerebellum and motor cortex during adaptive learning: the motor cortex retains what the cerebellum learns," Cerebral Cortex, vol. 21, no. 8, pp. 17611770, 2011.

[46] K. Ohtsuka and T. Enoki, "Transcranial magnetic stimulation over the posterior cerebellum during smooth pursuit eye movements in man," Brain, vol. 121, no. 3, pp. 429-435, 1998.

[47] M. Gerwig, F. P. Kolb, and D. Timmann, "The involvement of the human cerebellum in eyeblink conditioning," Cerebellum, vol. 6, no. 1, pp. 38-57, 2007.

[48] D. M. Wolpert, "Probabilistic models in human sensorimotor control," Human Movement Science, vol. 26, no. 4, pp. 511-524, 2007.

[49] K.-S. Tang, D. Kaski, J. H. J. Allum, and A. M. Bronstein, “The effect of gait approach velocity on the broken escalator phenomenon," Experimental Brain Research, vol. 226, no. 3, pp. 335346, 2013.

[50] Y.-H. Kim, S. H. You, M.-H. Ko et al., "Repetitive transcranial magnetic stimulation-induced corticomotor excitability and associated motor skill acquisition in chronic stroke," Stroke, vol. 37, no. 6, pp. 1471-1476, 2006.

[51] G. Ebersbach, C. Moreau, F. Gandor, L. Defebvre, and D. Devos, "Clinical syndromes: parkinsonian gait," Movement Disorders, vol. 28, no. 11, pp. 1552-1559, 2013.

[52] W. C. Koller, S. Glatt, B. Vetere-Overfield, and R. Hassanein, "Falls and Parkinson's disease," Clinical Neuropharmacology, vol. 12, no. 2, pp. 98-105, 1989.

[53] M. Weinrich, K. Koch, F. Garcia, and R. W. Angel, "Axial versus distal motor impairment in Parkinson's disease," Neurology, vol. 38, no. 4, pp. 540-545, 1988.

[54] H. L. Klawans, “Individual manifestations of Parkinson's disease after ten or more years of levodopa," Movement Disorders, vol. 1, no. 3, pp. 187-192, 1986.

[55] O. Blin, A. M. Ferrandez, J. Pailhous, and G. Serratrice, "Dopasensitive and dopa resistant gait parameters in Parkinson's disease," Journal of the Neurological Sciences, vol. 103, no. 1, pp. 51-54, 1991. 
[56] K. B. Foreman, M. L. Singer, O. Addison, R. L. Marcus, P. C. LaStayo, and L. E. Dibble, "Effects of dopamine replacement therapy on lower extremity kinetics and kinematics during a rapid force production task in persons with Parkinson disease," Gait and Posture, vol. 39, no. 1, pp. 638-640, 2014.

[57] R. T. Roemmich, N. Hack, U. Akbar, and C. J. Hass, "Effects of dopaminergic therapy on locomotor adaptation and adaptive learning in persons with Parkinson's disease," Behavioural Brain Research, vol. 268, pp. 31-39, 2014.

[58] E. D. Playford, I. H. Jenkins, R. E. Passingham, J. Nutt, R. S. J. Frackowiak, and D. J. Brooks, "Impaired mesial frontal and putamen activation in Parkinson's disease: a positron emission tomography study," Annals of Neurology, vol. 32, no. 2, pp. 151161, 1992.

[59] A. Pascual-Leone, J. Valls-Solé, J. P. Brasil-Neto, A. Cammarota, J. Grafman, and M. Hallett, "Akinesia in Parkinson's disease. II. Effects of subthreshold repetitive transcranial motor cortex stimulation," Neurology, vol. 44, no. 5, pp. 892-898, 1994.

[60] J. A. Williams, M. Imamura, and F. Fregni, "Updates of the use of non-invasive brain stimulation in physical and rehabilitation medicine," Journal of Rehabilitation Medicine, vol. 41, no. 5, pp. 305-311, 2009.

[61] G. Alon, D. A. Yungher, L. M. Shulman, and M. W. Rogers, "Safety and immediate effect of noninvasive transcranial pulsed current stimulation on gait and balance in parkinson disease," Neurorehabilitation and Neural Repair, vol. 26, no. 9, pp. 10891095, 2012.

[62] A. L. Hunt and K. D. Sethi, "The pull test: a history," Movement Disorders, vol. 21, no. 7, pp. 894-899, 2006.

[63] A. Lang, "Clinical rating scales and videotape analysis," in Therapy of Parkinson's Disease, W. C. Koller and G. Paulson, Eds., pp. 21-46, Marcel Dekker, New York, NY, USA, 1995.

[64] D. Kaski, R. Dominguez, J. Allum, A. Islam, and A. Bronstein, "Combining physical training with transcranial direct current stimulation to improve gait in Parkinson's disease: a pilot randomized controlled study," Clinical Rehabilitation, vol. 28, no. 11, pp. 1115-1124, 2014.

[65] B. R. Bloem, J. P. van Vugt, and D. J. Beckley, "Postural instability and falls in Parkinson's disease," Advances in Neurology, vol. 87, pp. 209-223, 2001.

[66] R. R. Pratt, "Art, dance, and music therapy," Physical Medicine \& Rehabilitation Clinics of North America, vol. 15, no. 4, pp. 827841, 2004.

[67] M. E. Hackney and G. M. Earhart, "Effects of dance on gait and balance in Parkinsons disease: a comparison of partnered and nonpartnered dance movement," Neurorehabilitation and Neural Repair, vol. 24, no. 4, pp. 384-392, 2010.

[68] K. Sacco, F. Cauda, L. Cerliani, D. Mate, S. Duca, and G. C. Geminiani, "Motor imagery of walking following training in locomotor attention. The effect of "the tango lesson"', NeuroImage, vol. 32, no. 3, pp. 1441-1449, 2006.

[69] D. Kaski, J. H. Allum, A. M. Bronstein, and R. O. Dominguez, "Applying anodal tDCS during tango dancing in a patient with Parkinson's disease," Neuroscience Letters, vol. 568, pp. 39-43, 2014.

[70] J. H. J. Allum and M. G. Carpenter, "A speedy solution for balance and gait analysis: angular velocity measured at the centre of body mass," Current Opinion in Neurology, vol. 18, no. 1, pp. 15-21, 2005.

[71] M. E. Tinetti, "Performance-orientated assessment of mobility problems in elderly patients," Journal of the American Geriatrics Society, vol. 34, no. 2, pp. 119-126, 1986.
[72] C. Geroin, A. Picelli, D. Munari, A. Waldner, C. Tomelleri, and N. Smania, "Combined transcranial direct current stimulation and robot-assisted gait training in patients with chronic stroke: a preliminary comparison," Clinical Rehabilitation, vol. 25, no. 6, pp. 537-548, 2011.

[73] S. Madhavan, K. A. Weber II, and J. W. Stinear, "Non-invasive brain stimulation enhances fine motor control of the hemiparetic ankle: implications for rehabilitation," Experimental Brain Research, vol. 209, no. 1, pp. 9-17, 2011.

[74] K. F. de Laat, A. G. W. van Norden, R. A. R. Gons et al., "Gait in elderly with cerebral small vessel disease," Stroke, vol. 41, no. 8, pp. 1652-1658, 2010.

[75] C. R. G. Guttmann, R. Benson, S. K. Warfield et al., "White matter abnormalities in mobility-impaired older persons," Neurology, vol. 54, no. 6, pp. 1277-1283, 2000.

[76] P. C. Gandiga, F. C. Hummel, and L. G. Cohen, "Transcranial DC stimulation (tDCS): a tool for double-blind shamcontrolled clinical studies in brain stimulation," Clinical Neurophysiology, vol. 117, no. 4, pp. 845-850, 2006.

[77] D. Kaski, R. O. Dominguez, J. H. Allum, and A. M. Bronstein, "Improving gait and balance in patients with leukoaraiosis using transcranial direct current stimulation and physical training: an exploratory study," Neurorehabilitation and Neural Repair, vol. 27, no. 9, pp. 864-871, 2013.

[78] M. Lotze, C. Braun, N. Birbaumer, S. Anders, and L. G. Cohen, "Motor learning elicited by voluntary drive," Brain, vol. 126, no. 4, pp. 866-872, 2003.

[79] J. M. Galea and P. Celnik, "Brain polarization enhances the formation and retention of motor memories," Journal of Neurophysiology, vol. 102, no. 1, pp. 294-301, 2009.

[80] M. A. Perez, B. K. Lungholt, K. Nyborg, and J. B. Nielsen, "Motor skill training induces changes in the excitability of the leg cortical area in healthy humans," Experimental Brain Research, vol. 159, no. 2, pp. 197-205, 2004.

[81] P. Celnik, N.-J. Paik, Y. Vandermeeren, M. Dimyan, and L. G. Cohen, "Effects of combined peripheral nerve stimulation and brain polarization on performance of a motor sequence task after chronic stroke," Stroke, vol. 40, no. 5, pp. 1764-1771, 2009.

[82] N. Roche, A. Lackmy, V. Achache, B. Bussel, and R. Katz, "Effects of anodal transcranial direct current stimulation over the leg motor area on lumbar spinal network excitability in healthy subjects," Journal of Physiology, vol. 589, no. 11, pp. 28132826, 2011.

[83] F. Fregni, P. S. Boggio, M. C. Santos et al., "Noninvasive cortical stimulation with transcranial direct current stimulation in Parkinson's disease," Movement Disorders, vol. 21, no. 10, pp. 1693-1702, 2006.

[84] D. P. Purpura and J. G. McMurtry, "Intracellular activities and evoked potential changes during polarization of motor cortex," Journal of Neurophysiology, vol. 28, pp. 166-185, 1965.

[85] H. Muramatsu and K. Koike, "Stroke rehabilitation therapy in a patient with a cardiac pacemaker for chronic atrial fibrillation," International Journal of Rehabilitation Research, vol. 26, no. 4, pp. 317-321, 2003.

[86] R. Dickstein, "Rehabilitation of gait speed after stroke: a critical review of intervention approaches," Neurorehabilitation and Neural Repair, vol. 22, no. 6, pp. 649-660, 2008.

[87] D.-Y. Kim, J.-Y. Lim, E. K. Kang et al., "Effect of transcranial direct current stimulation on motor recovery in patients with subacute stroke," The American Journal of Physical Medicine and Rehabilitation, vol. 89, no. 11, pp. 879-886, 2010. 
[88] R. Lindenberg, V. Renga, L. L. Zhu, D. Nair, and G. Schlaug, "Bihemispheric brain stimulation facilitates motor recovery in chronic stroke patients," Neurology, vol. 75, no. 24, pp. 2176$2184,2010$.

[89] G. Jayaram and J. W. Stinear, "The effects of transcranial stimulation on paretic lower limb motor excitability during walking," Journal of Clinical Neurophysiology, vol. 26, no. 4, pp. 272279, 2009.

[90] A. R. Brunoni, M. A. Nitsche, N. Bolognini et al., "Clinical research with transcranial direct current stimulation (tDCS): challenges and future directions," Brain Stimulation, vol. 5, no. 3, pp. 175-195, 2012.

[91] N. Murase, J. Duque, R. Mazzocchio, and L. G. Cohen, "Influence of interhemispheric interactions on motor function in chronic stroke," Annals of Neurology, vol. 55, no. 3, pp. 400-409, 2004.

[92] R. J. Nudo, "Recovery after brain injury: mechanisms and principles," Frontiers in Human Neuroscience, vol. 7, article 887, 2013.

[93] V. Tahtis, D. Kaski, and B. M. Seemungal, “The effect of single session bi-cephalic transcranial direct current stimulation on gait performance in sub-acute stroke: a pilot study," Restorative Neurology and Neuroscience, vol. 32, no. 4, pp. 527-532, 2014.

[94] C. Rossi, F. Sallustio, S. Di Legge, P. Stanzione, and G. Koch, "Transcranial direct current stimulation of the affected hemisphere does not accelerate recovery of acute stroke patients," European Journal of Neurology, vol. 20, no. 1, pp. 202-204, 2013. 

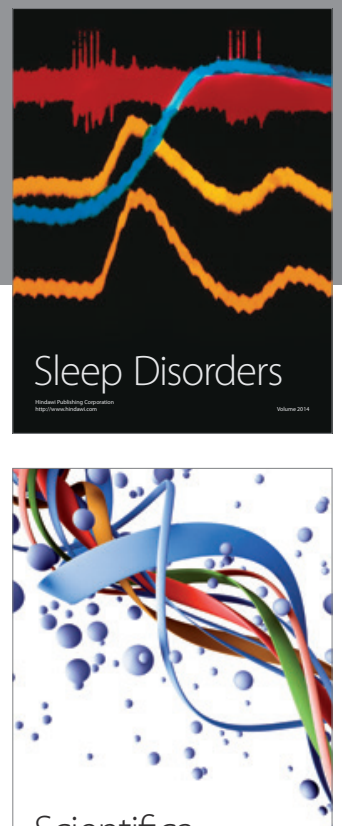

Scientifica
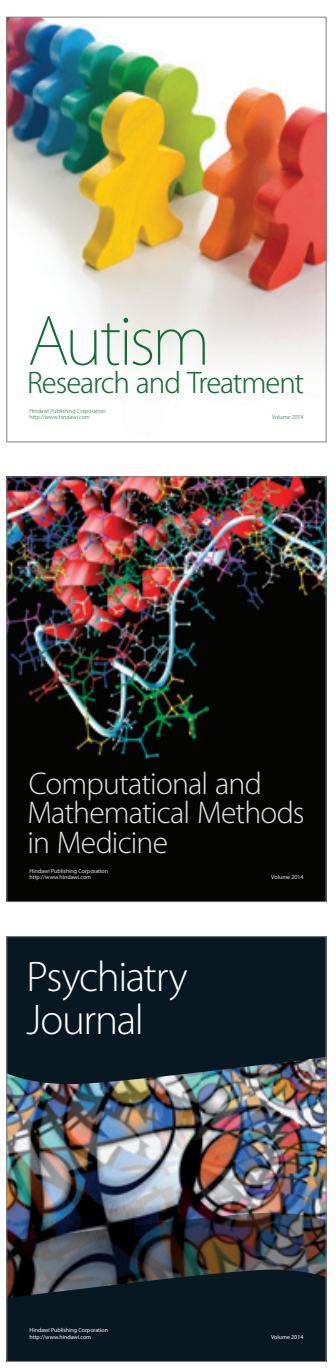
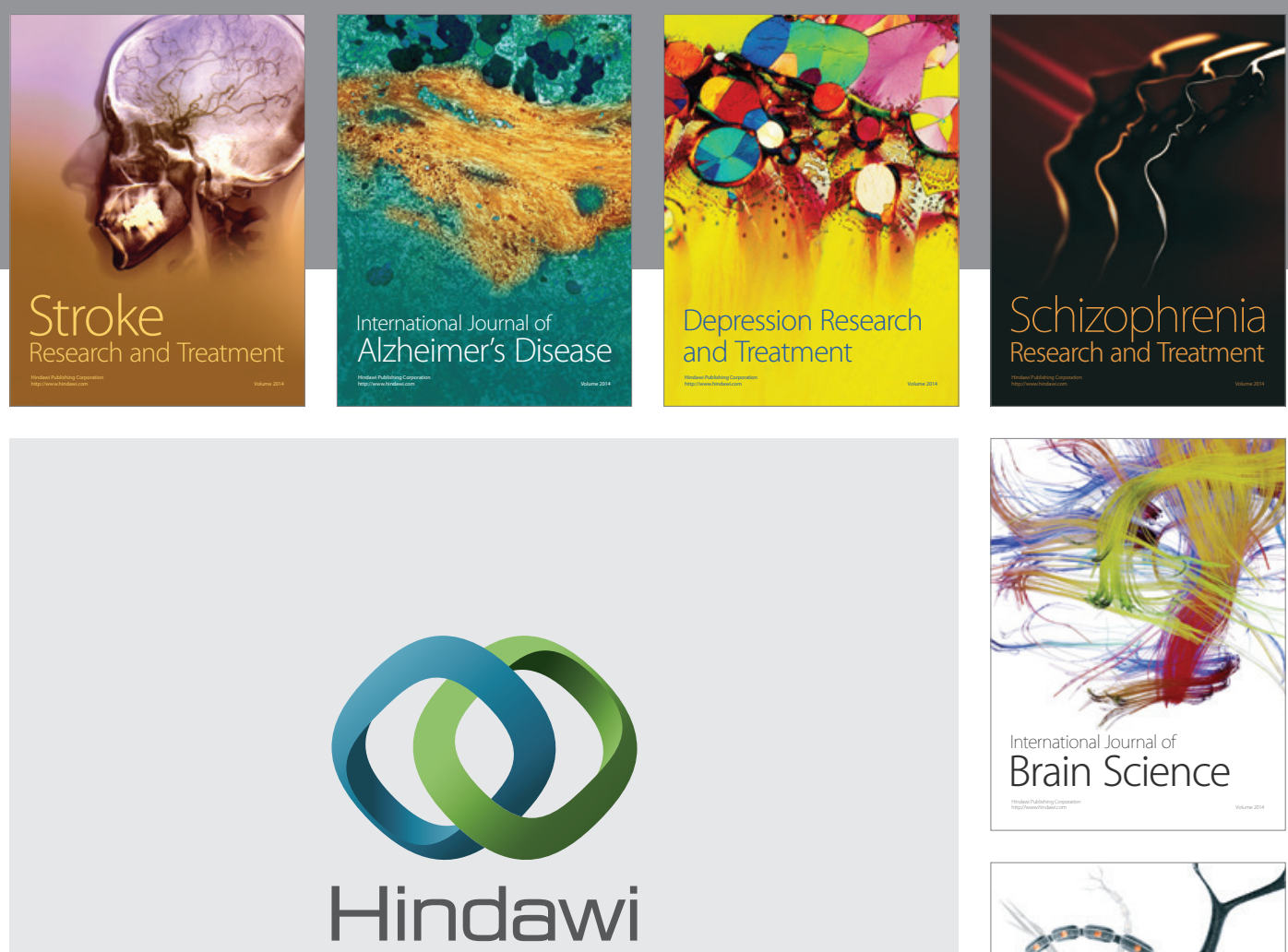

Submit your manuscripts at

http://www.hindawi.com
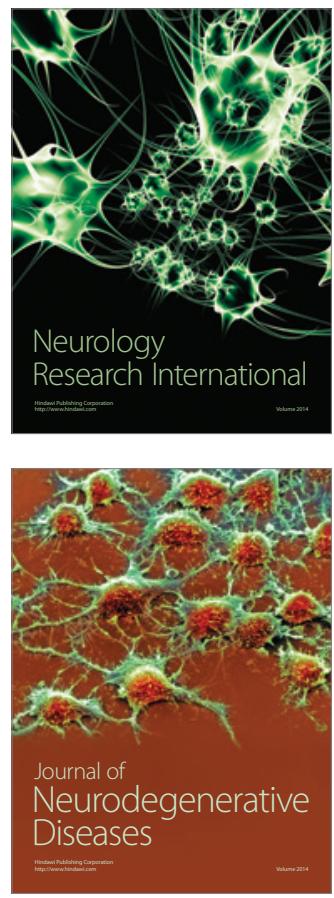

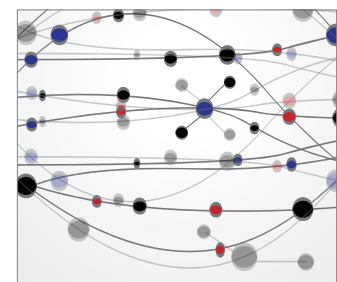

The Scientific World Journal
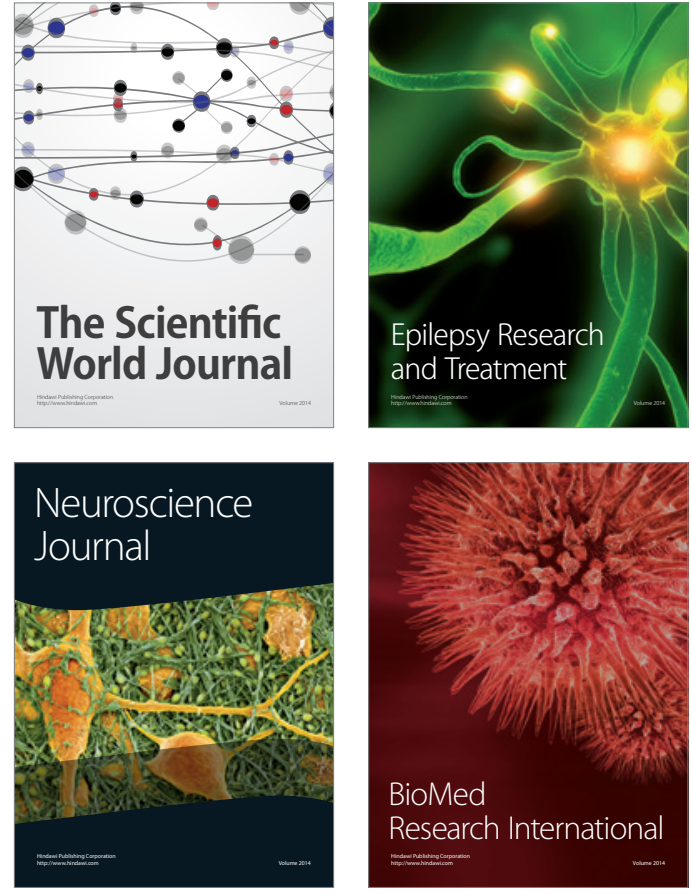

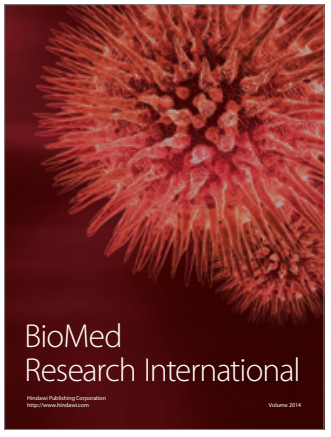

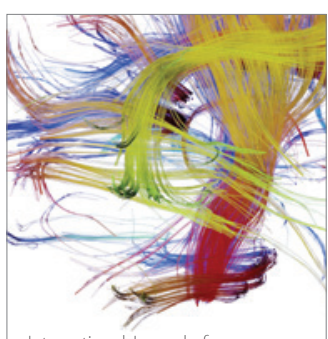

Brain Science

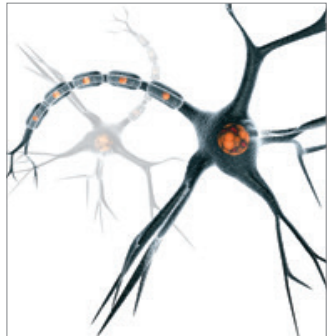

Neural Plasticity
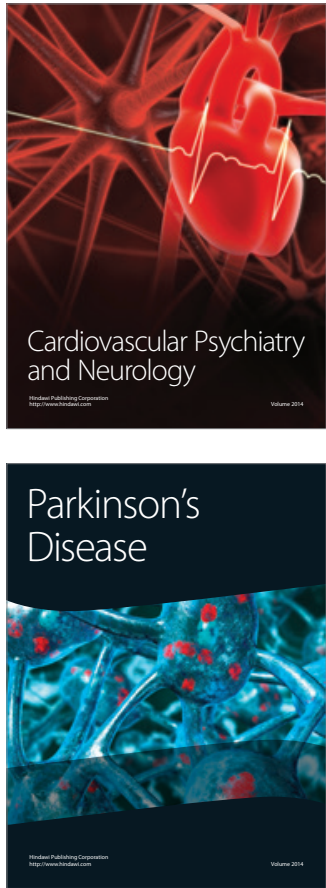